\title{
A Highly Regioselective Cyanothiolation of Alkynes via Oxidative Addition of Thiocyanates to Tetrakis(triphenylphosphine)palladium(0) Catalyst
}

\author{
Ikuyo Kamiya, ${ }^{\dagger}$ Jun-ichi Kawakami, ${ }^{\dagger}$ Shigenobu Yano, ${ }^{\dagger}$ Akihiro Nomoto, ${ }^{* \S}$ and Akiya Ogawa, ${ }^{*}$ \\ Division of material Science, Faculty of Science, Nara Women's University, Kitauoyanishi-machi, Nara \\ 630-8506, Japan, Takeda Pharmaceutical Company Limited, 2-17-85 Jusohonmachi, Yodogawa-ku, Osaka \\ 532-0024, Japan, and Department of Applied Chemistry, Faculty of Engineering, Osaka Prefecture University, \\ 1-1 Gakuen-cho, Sakai, Osaka 599-8531, Japan
}

\section{General Comments}

${ }^{1}$ H NMR spectra were recorded on JEOL JNM-GSX-270 (270 MHz), JEOL JNM-AL400 (400 MHz), and Varian GEMINI 2000 (300 MHz) spectrometers using $\mathrm{CDCl}_{3}$ as the solvent with $\mathrm{Me}_{4} \mathrm{Si}$ as the intermal standard.

${ }^{13} \mathrm{C}$ NMR spectra were taken on JEOL JNM-GSX-270 (68 MHz), JEOL JNM-GSX-400 (100 MHz), and Varian GEMINI 2000 (75 MHz) spectrometers using $\mathrm{CDCl}_{3}$ as the solvent. Chemical shifts in ${ }^{1} \mathrm{H}$ NMR were measured relative to $\mathrm{CDCl}_{3}$ and converted to $\delta(\mathrm{Me} \mathrm{Si})$ value by using $\delta\left(\mathrm{CDCl}_{3}\right)=7.26 \mathrm{ppm}$. Chemical shifts in ${ }^{13} \mathrm{C} \mathrm{NMR}$ were measured relative to $\mathrm{CDCl}_{3}$ and converted to $\delta\left(\mathrm{Me}_{4} \mathrm{Si}\right)$ value by using $\delta\left(\mathrm{CDCl}_{3}\right)=77.0 \mathrm{ppm}$. IR spectra were determined on a Perkin Elmer Model 1600 spectrometer or JUSCO FT/IR-8900 $\mu$ Fourier Transform Infrared Microsampling System. Mass spectra were obtained on JEOL JMS-DX303 in the analytical section of Osaka University. Elemental analyses were performed in the analytical section of there. All materials were obtained from commercial supplies and purified by distillation or recrystallization. Thiocyanates were prepared as described in the literature (see: D. N. Harpp, B.T. Friedlander, R. A. Smith, Synthesis 1979, 181). 


\section{Experimental Section}

\section{General Procedure}

Representative procedure: Method A (the cyanothiolation in solvent, entries 1-3 and 8 in Table 2): Into a 50 $\mathrm{mL}$ stainless steel autoclave with a magnetic stirring bar under an argon atmosphere were placed $\mathrm{Pd}\left(\mathrm{PPh}_{3}\right)_{4}(10$ $\mathrm{mol} \%)$, benzene $(1 \mathrm{~mL})$, alkyne $(1.0 \mathrm{mmol})$, and thiocyanate $(1.0 \mathrm{mmol})$. Argon gas was purged three times and then charged at $0.1 \mathrm{MPa}$. The reaction was conducted with magnetic stirring for $66 \mathrm{~h}$ upon heating at $120{ }^{\circ} \mathrm{C}$. Then, the resulting mixture was filtered through Celite and concentrated in vacuo. Purification of the product was carried out by MPLC (silica gel, $25-40 \mu \mathrm{m}$, length $310 \mathrm{~mm}$, i.d. $25 \mathrm{~mm}$, eluent $n$-hexane/ $\mathrm{Et}_{2} \mathrm{O}$ ) or silica gel column chromatography.

Method B (the cyanothiolation in the absence of solvent, entries 4-7 in Table 2): Into a flame-dried two-necked flask $(20 \mathrm{~mL})$ with a reflux condenser and a magnetic stirring bar under a nitrogen atmosphere were placed $\mathrm{Pd}\left(\mathrm{PPh}_{3}\right)_{4}(10 \mathrm{~mol} \%)$, alkyne $(1.0 \mathrm{mmol})$, and thiocyanate $(1.0 \mathrm{mmol})$. The reaction was conducted with magnetic stirring for $66 \mathrm{~h}$ upon heating at $120^{\circ} \mathrm{C}$, and then the resulting mixture was filtered through Celite and concentrated in vacuo. Purification of the product was carried out by using a recycling preparative HPLC (Japan Analytical Industry Co. Ltd., Model LC-908) equipped with JAIGEL-1H and -2H columns (GPC) using $\mathrm{CHCl}_{3}$ as an eluent or preparative TLC (PTLC) on Wakogel B-5F silica gel (eluent $n$-hexane/Et $2 \mathrm{O}$ ).

\section{Spectral and Analytical Data}

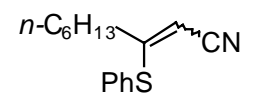

[(2Z)-3-Phenylthionon-2-enenitrile (Table 2, entry 1)]: Pale red-brown oil.

${ }^{1} \mathrm{H}$ NMR $\left(270 \mathrm{MHz}, \mathrm{CDCl}_{3}, 25^{\circ} \mathrm{C}, \mathrm{TMS}\right): \delta=0.83\left(\mathrm{t},{ }^{3} J(\mathrm{H}, \mathrm{H})=6.8 \mathrm{~Hz}, 3 \mathrm{H}\right), 1.14-1.25(\mathrm{~m}, 6 \mathrm{H}), 1.39$ (quint, ${ }^{3} J$ $(\mathrm{H}, \mathrm{H})=7.3 \mathrm{~Hz}, 2 \mathrm{H}), 2.15\left(\mathrm{t},{ }^{3} J(\mathrm{H}, \mathrm{H})=7.6 \mathrm{~Hz}, 2 \mathrm{H}\right), 5.30(\mathrm{~s}, 1 \mathrm{H}), 7.38-7.51(\mathrm{~m}, 5 \mathrm{H}) ;{ }^{13} \mathrm{C} \mathrm{NMR}(68 \mathrm{MHz}$, $\left.\mathrm{CDCl}_{3}, 25^{\circ} \mathrm{C}, \mathrm{TMS}\right): \delta=13.94,22.35,28.24,28.27,31.25,36.24,93.67,116.08,129.39,129.48,129.57,134.89$, 165.51; IR (NaCl) 3060, 2958, 2929, 2857, 2212 (CN), 1574, 1441, 1069, 1024, 749, 705, $692 \mathrm{~cm}^{-1}$; MS (EI): $\mathrm{m} / z$ (\%): 245 (20) $\left[\mathrm{M}^{+}\right]$. Anal. Calcd for $\mathrm{C}_{15} \mathrm{H}_{19} \mathrm{NS}: \mathrm{C}, 73.42 ; \mathrm{H}, 7.80 ; \mathrm{N}, 5.71$. Found: C, 73.16; H, 7.94; N, 5.25. NOE experiment: irradiation of the $\mathrm{C}-4$ methylene triplet at $\delta 2.15$ resulted in a enhancement of the signal at $\delta$ 5.30 (vinyl singlet). 
[(2E)-3-Phenylthionon-2-enenitrile (Table 2, entry 1)]: Pale red-brown oil.

${ }^{1} \mathrm{H} \mathrm{NMR}\left(270 \mathrm{MHz}, \mathrm{CDCl}_{3}, 25^{\circ} \mathrm{C}, \mathrm{TMS}\right): \delta=0.91\left(\mathrm{t},{ }^{3} J(\mathrm{H}, \mathrm{H})=6.6 \mathrm{~Hz}, 3 \mathrm{H}\right), 1.30-1.46(\mathrm{~m}, 6 \mathrm{H}), 1.71$ (quint, ${ }^{3} J$ $(\mathrm{H}, \mathrm{H})=7.4 \mathrm{~Hz}, 2 \mathrm{H}), 2.63\left(\mathrm{t},{ }^{3} J(\mathrm{H}, \mathrm{H})=7.8 \mathrm{~Hz}, 2 \mathrm{H}\right), 4.46(\mathrm{~s}, 1 \mathrm{H}), 7.47-7.52(\mathrm{~m}, 5 \mathrm{H}) ;{ }^{13} \mathrm{C} \mathrm{NMR}(68 \mathrm{MHz}$, $\left.\mathrm{CDCl}_{3}, 25^{\circ} \mathrm{C}, \mathrm{TMS}\right): \delta=14.03,22.51,28.65,29.17,31.43,35.50,89.36,116.85,128.64,130.12,130.43,135.55$, 169.41; IR (NaCl) 3057, 2956, 2929, 2858, 2210 (CN), 1579, 1476, 1466, 1441, 1068, 1024, 790, 751, 705, 691 $\mathrm{cm}^{-1}$; MS (EI): $m / z(\%): 245$ (16) $\left[\mathrm{M}^{+}\right]$. Anal. Calcd for $\mathrm{C}_{15} \mathrm{H}_{19} \mathrm{NS}: \mathrm{C}, 73.42 ; \mathrm{H}, 7.80 ; \mathrm{N}, 5.71$. Found: $\mathrm{C}$, $73.19 ; \mathrm{H}, 7.80 ; \mathrm{N}, 5.43$.

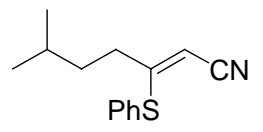

[(2Z)-6-Methyl-3-phenylthiohept-2-enenitrile (Table 2, entry 2)]: Brown oil.

${ }^{1} \mathrm{H} N M R\left(300 \mathrm{MHz}, \mathrm{CDCl}_{3}, 25^{\circ} \mathrm{C}, \mathrm{TMS}\right): \delta=0.72\left(\mathrm{~d},{ }^{3} J(\mathrm{H}, \mathrm{H})=6.2 \mathrm{~Hz}, 6 \mathrm{H}\right), 1.25-1.40(\mathrm{~m}, 3 \mathrm{H}), 2.15\left(\mathrm{t},{ }^{3} J(\mathrm{H}\right.$, $\mathrm{H})=7.6 \mathrm{~Hz}, 2 \mathrm{H}), 5.30(\mathrm{~s}, 1 \mathrm{H}), 7.38-7.52(\mathrm{~m}, 5 \mathrm{H}) ;{ }^{13} \mathrm{C} \mathrm{NMR}\left(68 \mathrm{MHz}, \mathrm{CDCl}_{3}, 25{ }^{\circ} \mathrm{C}, \mathrm{TMS}\right): \delta=22.04,27.34$, 34.16, 37.50, 93.30, 116.09, 129.27, 129.33, 129.58, 134.93, 165.94; IR (NaCl) 3059, 2957, 2930, 2869, 2211 (CN), 1573, 1441, 1386, 1368, 1309, 1082, 1069, 1024, 749, 706, $692 \mathrm{~cm}^{-1}$; MS (EI): $m / z(\%): 231$ (8.5) [M']. Anal. Calcd for $\mathrm{C}_{14} \mathrm{H}_{17} \mathrm{NS}$ : C, 72.33; H, 7.41; N, 6.05. Found: C, 72.33; H, 7.39; N, 5.99. NOE experiment: irradiation of the $\mathrm{C}-4$ methylene triplet at $\delta 2.15$ resulted in a enhancement of the signal at $\delta 5.30$ (vinyl singlet).

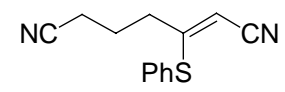

[(2Z)-3-Phenylthiohept-5-enedinitrile (Table 2, entry 3)]: Brown oil.

${ }^{1} \mathrm{H} \mathrm{NMR}\left(300 \mathrm{MHz}, \mathrm{CDCl}_{3}, 25{ }^{\circ} \mathrm{C}, \mathrm{TMS}\right): \delta=1.76$ (quint, ${ }^{3} \mathrm{~J}(\mathrm{H}, \mathrm{H})=7.0 \mathrm{~Hz}, 2 \mathrm{H}$ ), $2.25\left(\mathrm{t},{ }^{3} J(\mathrm{H}, \mathrm{H})=7.2 \mathrm{~Hz}\right.$, $2 \mathrm{H}), 2.36\left(\mathrm{t},{ }^{3} J(\mathrm{H}, \mathrm{H})=7.3 \mathrm{~Hz}, 2 \mathrm{H}\right), 5.41(\mathrm{~s}, 1 \mathrm{H}), 7.35-7.52(\mathrm{~m}, 5 \mathrm{H}) ;{ }^{13} \mathrm{C} \mathrm{NMR}\left(68 \mathrm{MHz}, \mathrm{CDCl}_{3}, 25^{\circ} \mathrm{C}, \mathrm{TMS}\right)$ : $\delta=16.08,23.81,34.52,95.83,115.40,118.35,128.39,129.71,129.99,134.64,162.29 ; \mathrm{IR}(\mathrm{NaCl}) 3048,2943$, 2246 (CN), 2212 (CN), 1573, 1475, 1455, 1441, 1424, 1024, 751, 705, $692 \mathrm{~cm}^{-1}$; MS (EI): $m / z$ (\%): 229 (8.6) $\left[\mathrm{M}^{+}\right]$. HRMS calcd for $\mathrm{C}_{13} \mathrm{H}_{12} \mathrm{~N}_{2} \mathrm{~S}$ 229.0799, found 229.0809. NOE experiment: irradiation of the C-4 methylene triplet at $\delta 2.36$ resulted in a enhancement of the signal at $\delta 5.41$ (vinyl singlet). 


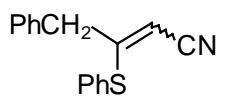

[4-Phenyl-3-phenylthiopent-2-enenitrile [E/Z-mixture] (Table 2, entry 4)]: Pale yellow oil.

${ }^{1} \mathrm{H}$ NMR (300 MHz, $\left.\mathrm{CDCl}_{3}, 25^{\circ} \mathrm{C}, \mathrm{TMS}\right): \delta=3.42\left(\mathrm{t},{ }^{4} J(\mathrm{H}, \mathrm{H})=0.75,2 \mathrm{H}\right), 3.95(\mathrm{~s}, 2 \mathrm{H}), 4.58(\mathrm{~s}, 1 \mathrm{H}), 5.11\left(\mathrm{t},{ }^{3} J\right.$ $(\mathrm{H}, \mathrm{H})=1.4,1 \mathrm{H}), 6.93-7.49(\mathrm{~m}, 20 \mathrm{H}) ;{ }^{13} \mathrm{C} \mathrm{NMR}\left(75 \mathrm{MHz}, \mathrm{CDCl}_{3}, 25^{\circ} \mathrm{C}, \mathrm{TMS}\right): \delta=42.22,77.18,77.23,95.18$, 95.27, 116.02, 127.40, 128.79, 128.92, 129.09, 129.50, 129.89, 135.39, 135.73, 164.30; IR (NaCl) 3061, 3028, 2924, 2210 (CN), 1574, 1495, 1474, 1454, 1441, 1069, 1024, 783, $692 \mathrm{~cm}^{-1}$; MS (EI): m/z (\%): 251 (23) [M]. HRMS calcd for $\mathrm{C}_{16} \mathrm{H}_{13} \mathrm{NS} 251.0770$, found 251.0768.

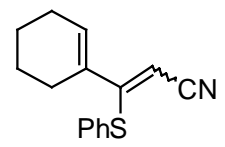

[(2Z)-3-Cyclohex-1-enyl-3-phenylthioprop-2-enenitrile (Table 2, entry 5)]: Pale yellow oil.

${ }^{1} \mathrm{H}$ NMR (300 MHz, $\left.\mathrm{CDCl}_{3}, 25^{\circ} \mathrm{C}, \mathrm{TMS}\right): \delta=1.43-1.72(\mathrm{~m}, 4 \mathrm{H}), 2.16-2.22(\mathrm{~m}, 2 \mathrm{H}), 2.30-2.36(\mathrm{~m}, 2 \mathrm{H}), 4.54$ (s, 1H), 6.24 (sept like, $\left.{ }^{5} J(\mathrm{H}, \mathrm{H})=1.8 \mathrm{~Hz}, 1 \mathrm{H}\right), 7.42-7.50(\mathrm{~m}, 5 \mathrm{H}) ;{ }^{13} \mathrm{C} \mathrm{NMR}\left(75 \mathrm{MHz}, \mathrm{CDCl}_{3}, 25{ }^{\circ} \mathrm{C}, \mathrm{TMS}\right): \delta=$ 21.27, 22.24, 25.34, 27.72, 88.00, 117.41, 129.50, 130.03, 130.23, 132.98, 134.01, 135.31, 168.84; IR (NaCl) 3036, 2932, 2858, 2835, $2208(\mathrm{C} \equiv \mathrm{N}), 1558,1475,1441,748,706 \mathrm{~cm}^{-1}$; MS (EI): $m / z(\%): 241(36)\left[\mathrm{M}^{+}\right]$. Anal. Calcd for $\mathrm{C}_{15} \mathrm{H}_{15} \mathrm{NS}$ : C, 74.65; H, 6.26; N, 5.80. Found: $\mathrm{C}, 74.70 ; \mathrm{H}, 6.55 ; \mathrm{N}, 5.29$. HRMS calcd for $\mathrm{C}_{15} \mathrm{H}_{15} \mathrm{NS}$ 241.0927, found 241.0921. NOE experiment: irradiation of the C-4 vinyl singlet at $\delta 4.54$ resulted in a enhancement of the signal at $\delta 6.24$ (vinyl singlet).

[(2E)-3-Cyclohex-1-enyl-3-phenylthioprop-2-enenitrile (Table 2, entry 5)]: Yellow oil.

${ }^{1} \mathrm{H} N M R\left(300 \mathrm{MHz}, \mathrm{CDCl}_{3}, 25^{\circ} \mathrm{C}, \mathrm{TMS}\right): \delta=1.47-1.50$ (m, 4H), 2.05-2.09 (m, 4H), $5.56(\mathrm{~s}, 1 \mathrm{H}), 6.46\left(\mathrm{t},{ }^{3} J(\mathrm{H}\right.$, $\mathrm{H})=4.1 \mathrm{~Hz}, 1 \mathrm{H}), 7.27-7.32(\mathrm{~m}, 5 \mathrm{H}) ;{ }^{13} \mathrm{C} \mathrm{NMR}\left(75 \mathrm{MHz}, \mathrm{CDCl}_{3}, 25{ }^{\circ} \mathrm{C}, \mathrm{TMS}\right): \delta=21.67,22.06,25.96,26.90$, 96.74, 116.88, 127.77, 129.09, 131.34, 133.20, 134.60, 136.47, 161.45; IR (NaCl) 3048, 2932, 2860, 2361, 2210(CN), 1555, 1479, 1458, 745, $689 \mathrm{~cm}^{-1}$; MS (EI): $\mathrm{m} / z$ (\%): 241 (110) $\left[\mathrm{M}^{+}\right]$. HRMS calcd for $\mathrm{C}_{15} \mathrm{H}_{15} \mathrm{NS}$ 241.0927, found 241.0921.

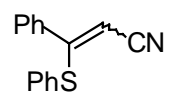

[(2Z)-3-Phenyl-3-phenylthioprop-2-enenitrile (Table 2, entry 6)]: Pale yellow oil. 
${ }^{1} \mathrm{H}$ NMR $\left(300 \mathrm{MHz}, \mathrm{CDCl}_{3}, 25{ }^{\circ} \mathrm{C}, \mathrm{TMS}\right): \delta=5.65(\mathrm{~s}, 1 \mathrm{H}), 7.16-7.44(\mathrm{~m}, 10 \mathrm{H}) ;{ }^{13} \mathrm{C} \mathrm{NMR}\left(75 \mathrm{MHz}, \mathrm{CDCl}_{3}\right.$, $\left.25^{\circ} \mathrm{C}, \mathrm{TMS}\right): \delta=77.19$ 97.19, 128.33, 128.45, 128.64, 129.14, 130.58, 131,36, 132.59, 136.25, 161.60; IR $(\mathrm{NaCl})$ 2924, 2854, $2210(\mathrm{C} \equiv \mathrm{N}), 1556,1441,745,691 \mathrm{~cm}^{-1}$; MS (EI): $m / z(\%): 237$ (22) [M']. HRMS calcd for $\mathrm{C}_{15} \mathrm{H}_{11} \mathrm{NS}$ 237.0613, found 237.0609.

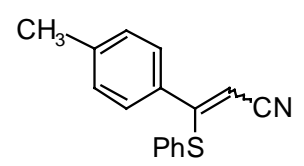

[3-(4-methyphenyl)-3-phenylthioprop-2-enenitrile [E/Z-mixture] (Table 2, entry 7)]: Deep yellow oil.

${ }^{1} \mathrm{H} \mathrm{NMR}\left(300 \mathrm{MHz}, \mathrm{CDCl}_{3}, 25^{\circ} \mathrm{C}, \mathrm{TMS}\right): \delta=2.29$ (s, 3H), 2.40 (s, 3H), 4.70 (s, 1H), 5.64 (s, 1H), 7.05-7.59 (m, $18 \mathrm{H}) ;{ }^{13} \mathrm{C} \mathrm{NMR}\left(75 \mathrm{MHz}, \mathrm{CDCl}_{3}, 25{ }^{\circ} \mathrm{C}, \mathrm{TMS}\right): \delta=21.17,21.33,89.09,96.74,116.60,117.56,128.20,128.35$, 129.13, 129.39, 129.58, 130.29, 130.57, 131.76, 132.30, 133.38, 135.44, 141.16, 141.34, 161.16, 166.22; IR (NaCl) 3055, 3020, 2918, $2156(\mathrm{C} \equiv \mathrm{N}), 1578,1475,1439,810,737,687 \mathrm{~cm}^{-1}$; MS (EI): $m / z(\%): 251(13)\left[\mathrm{M}^{+}\right]$. HRMS calcd for $\mathrm{C}_{16} \mathrm{H}_{13} \mathrm{NS} 251.0770$, found 251.0767. 
${ }^{13} \mathrm{C}-{ }^{13} \mathrm{C}$ COSY Spectrum (INADEQUATE)

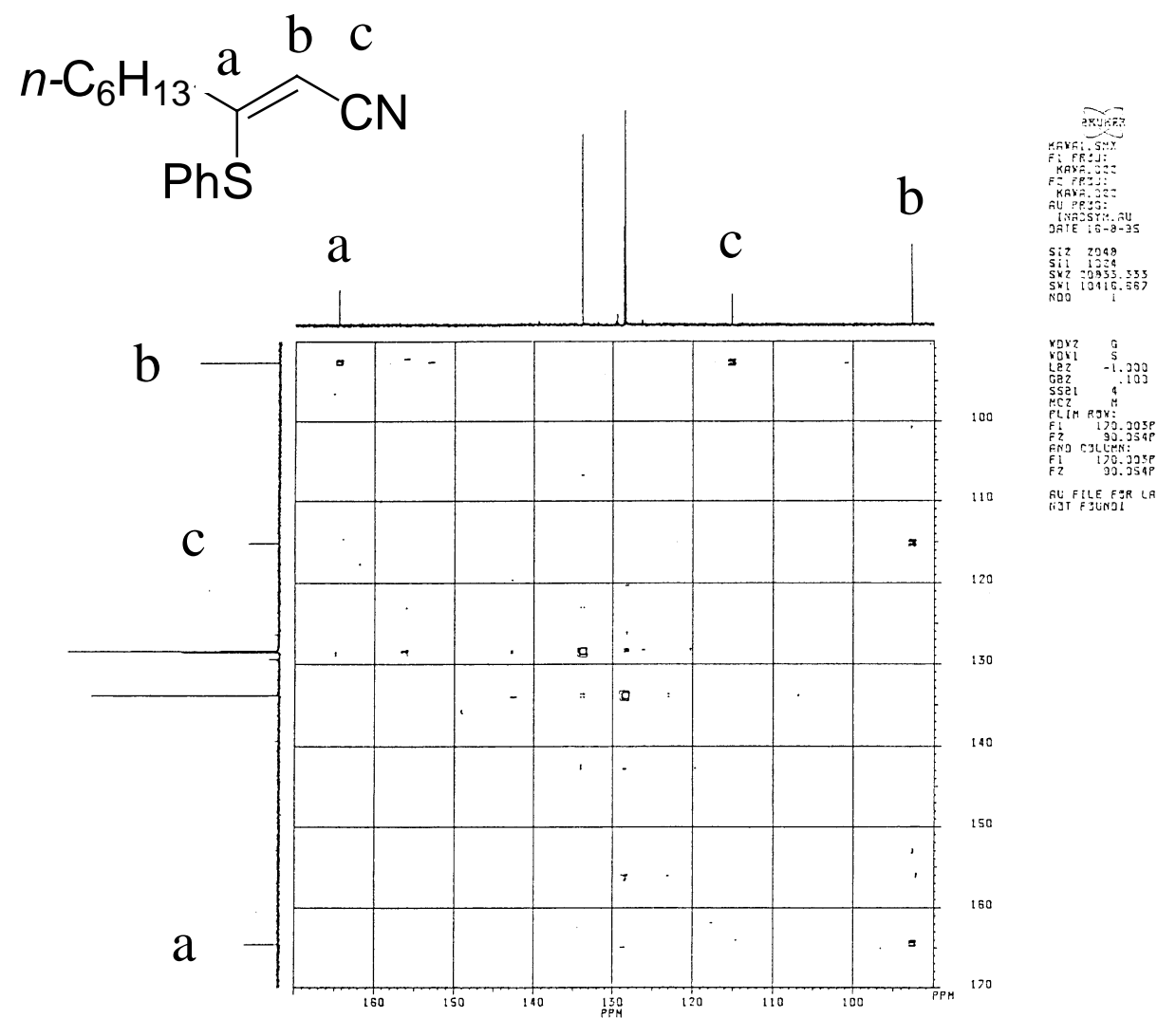



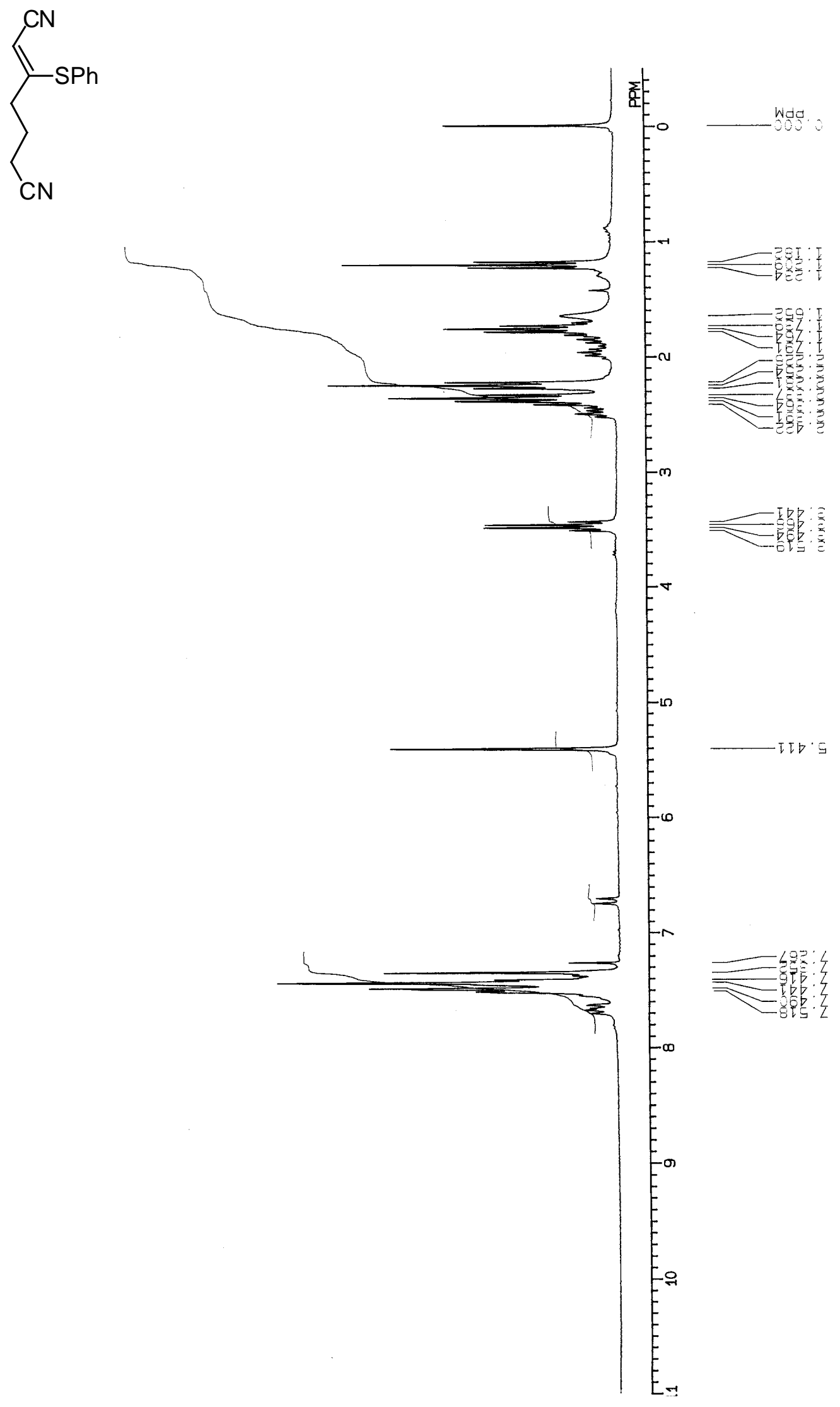


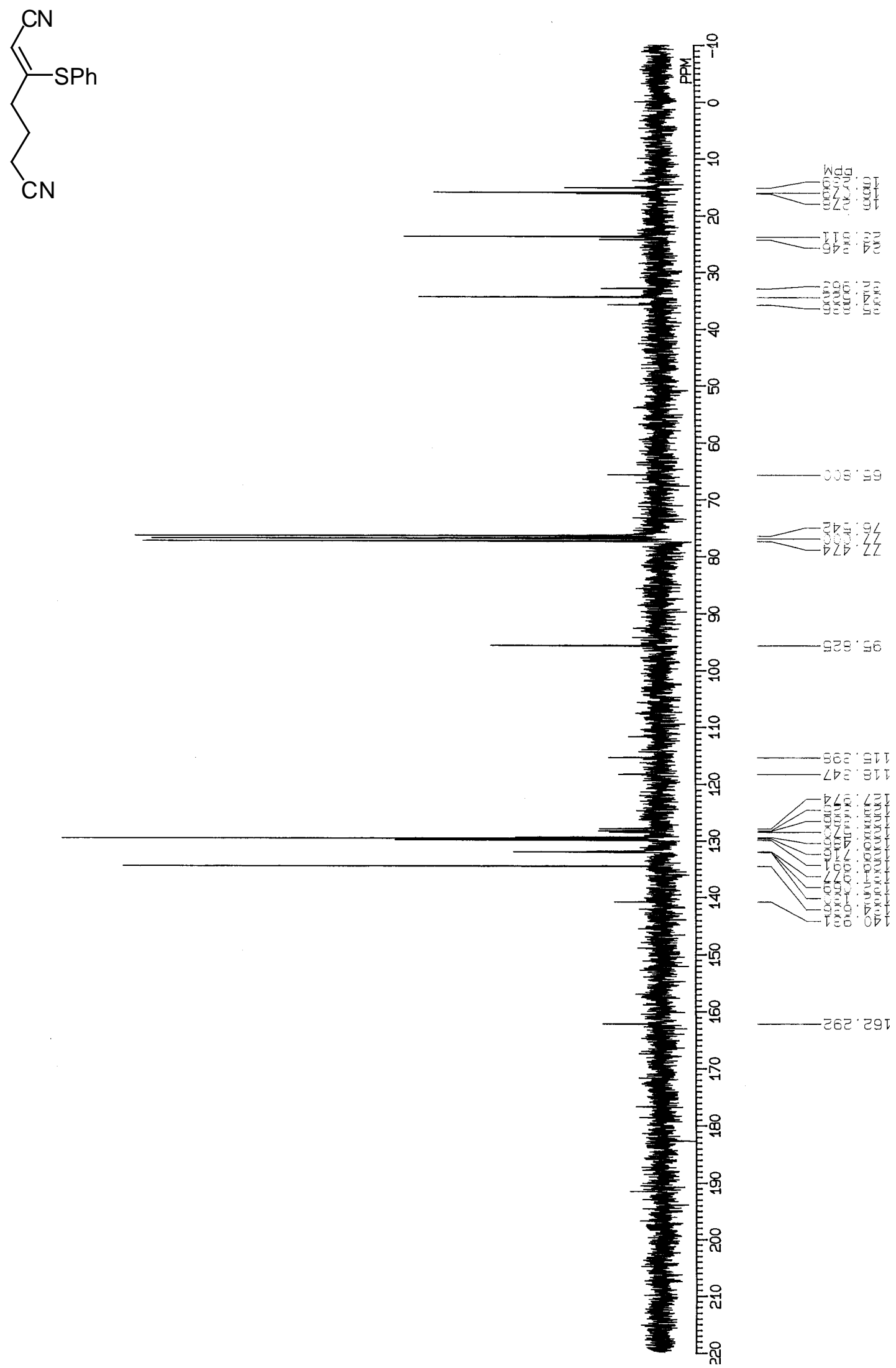




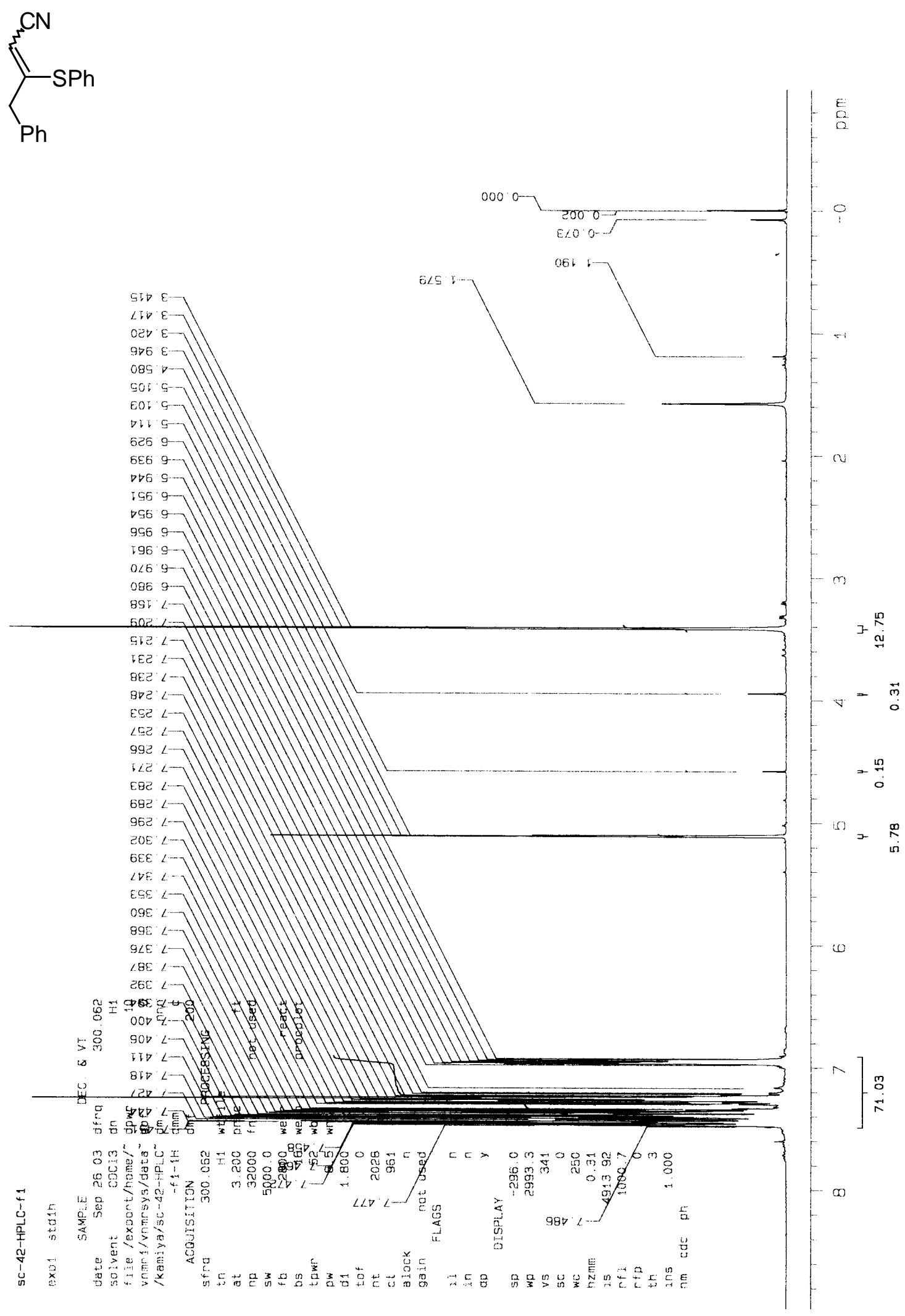




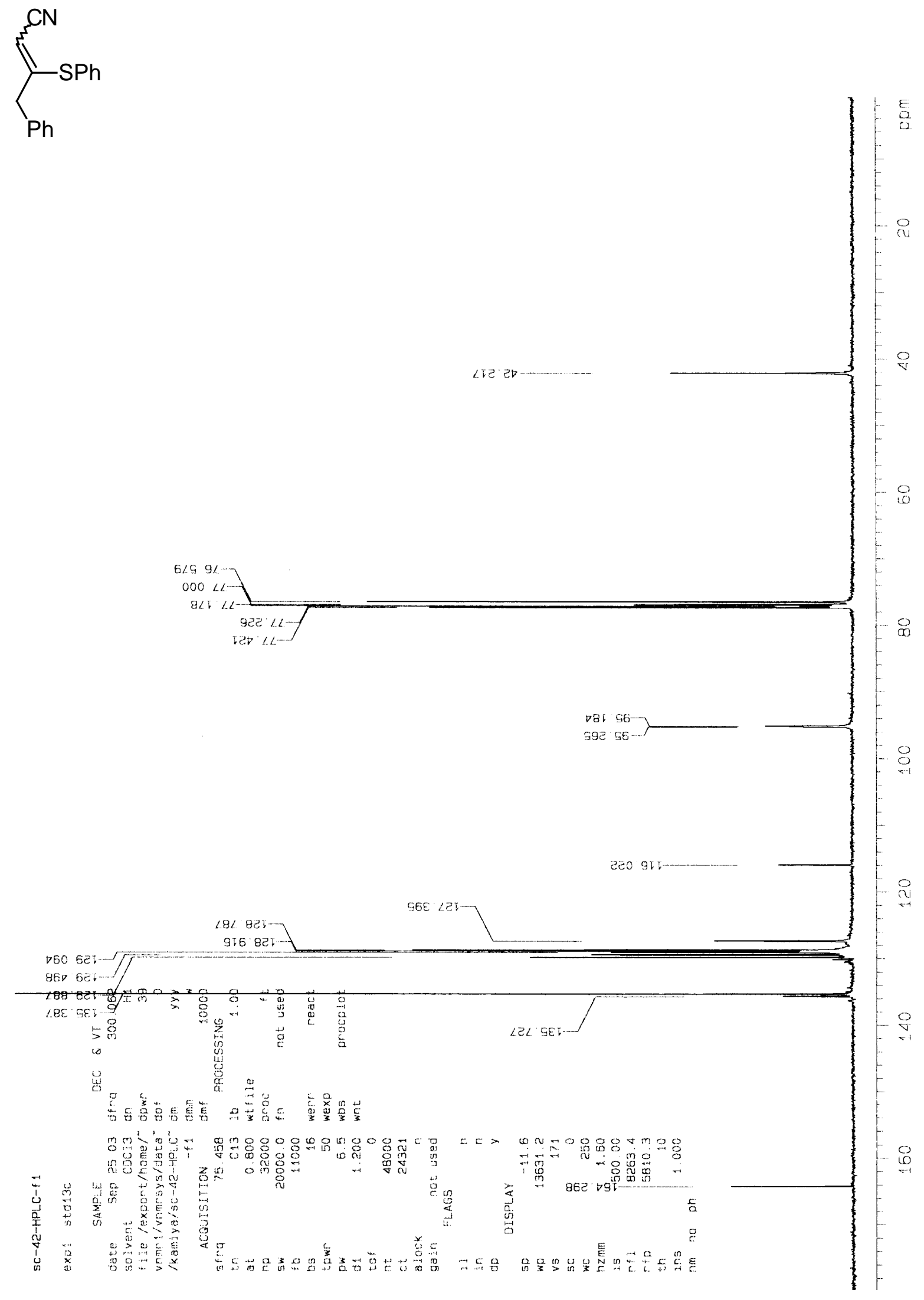



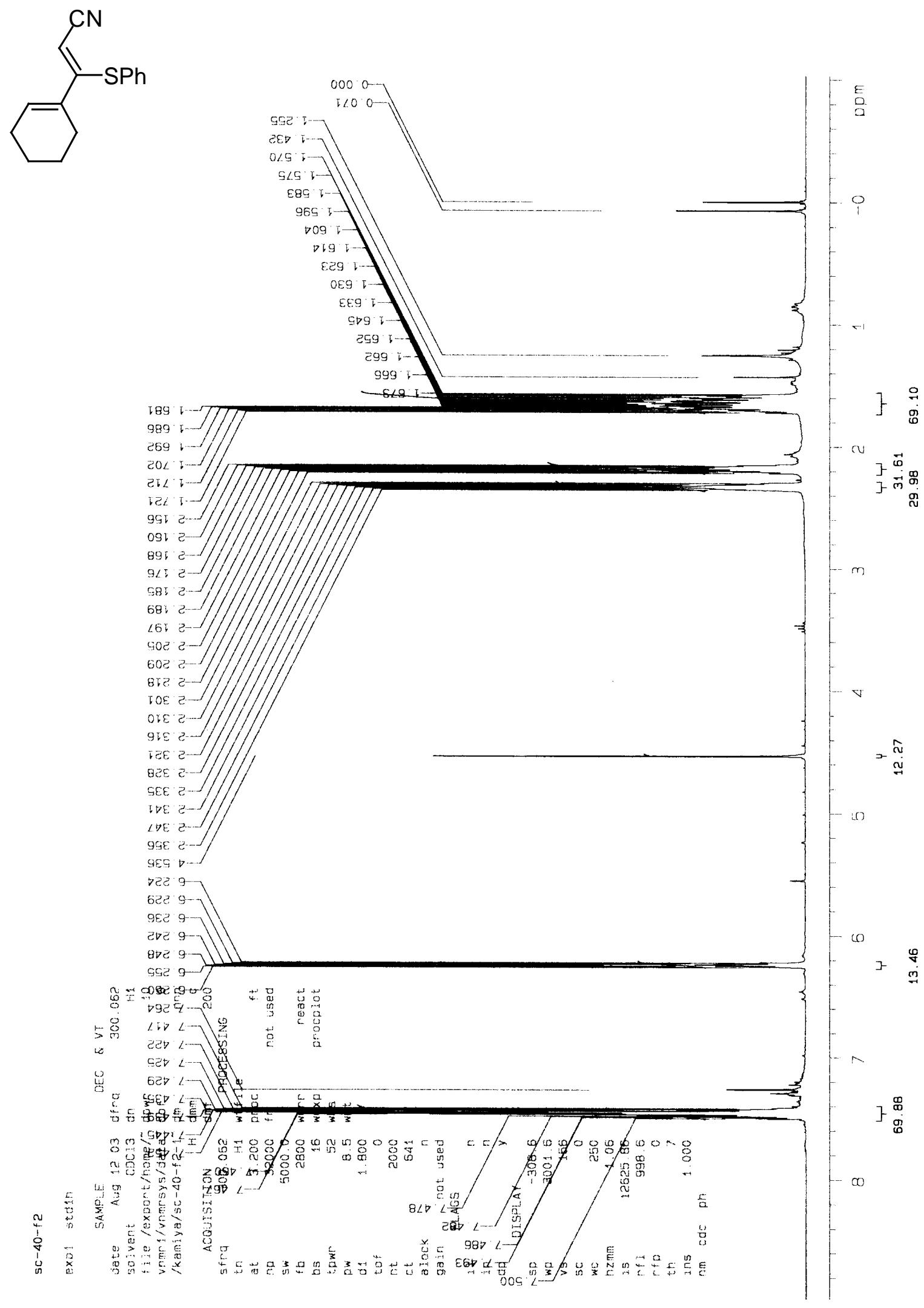

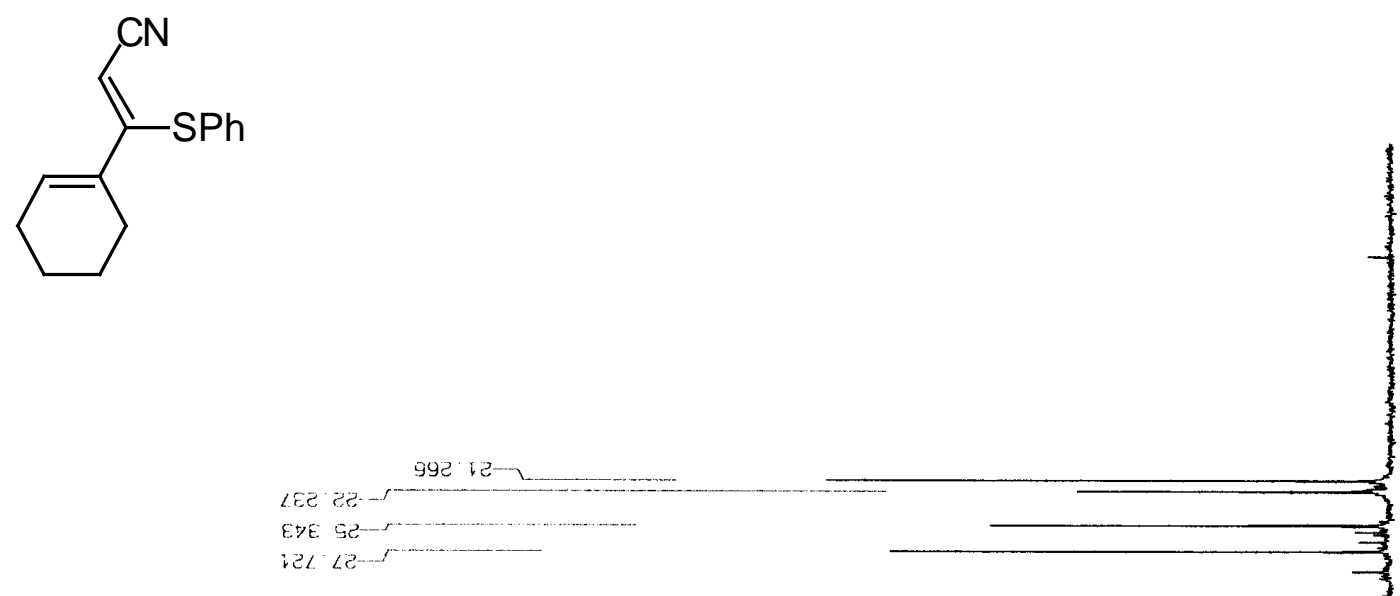

$6 / 99$ $000<1<$ $120+11, \cdots$

टEO OE,

Gटट OE:...

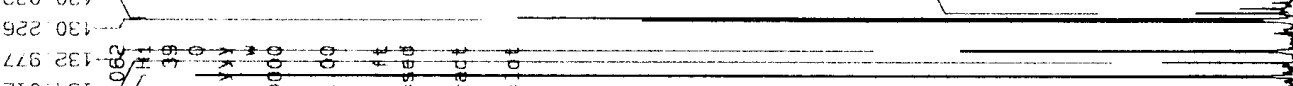

Z:O DE F...

जoe $9 \varepsilon^{2} \mathrm{~s}$

$\omega$

岁

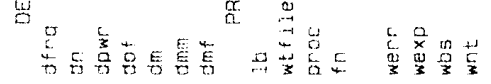

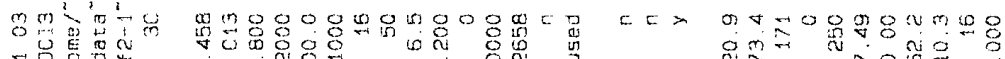

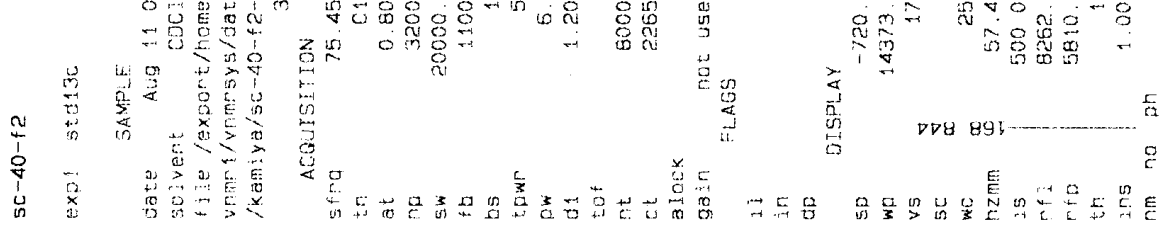

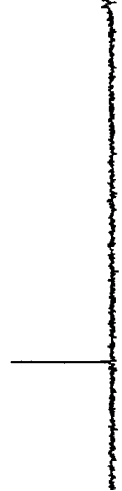




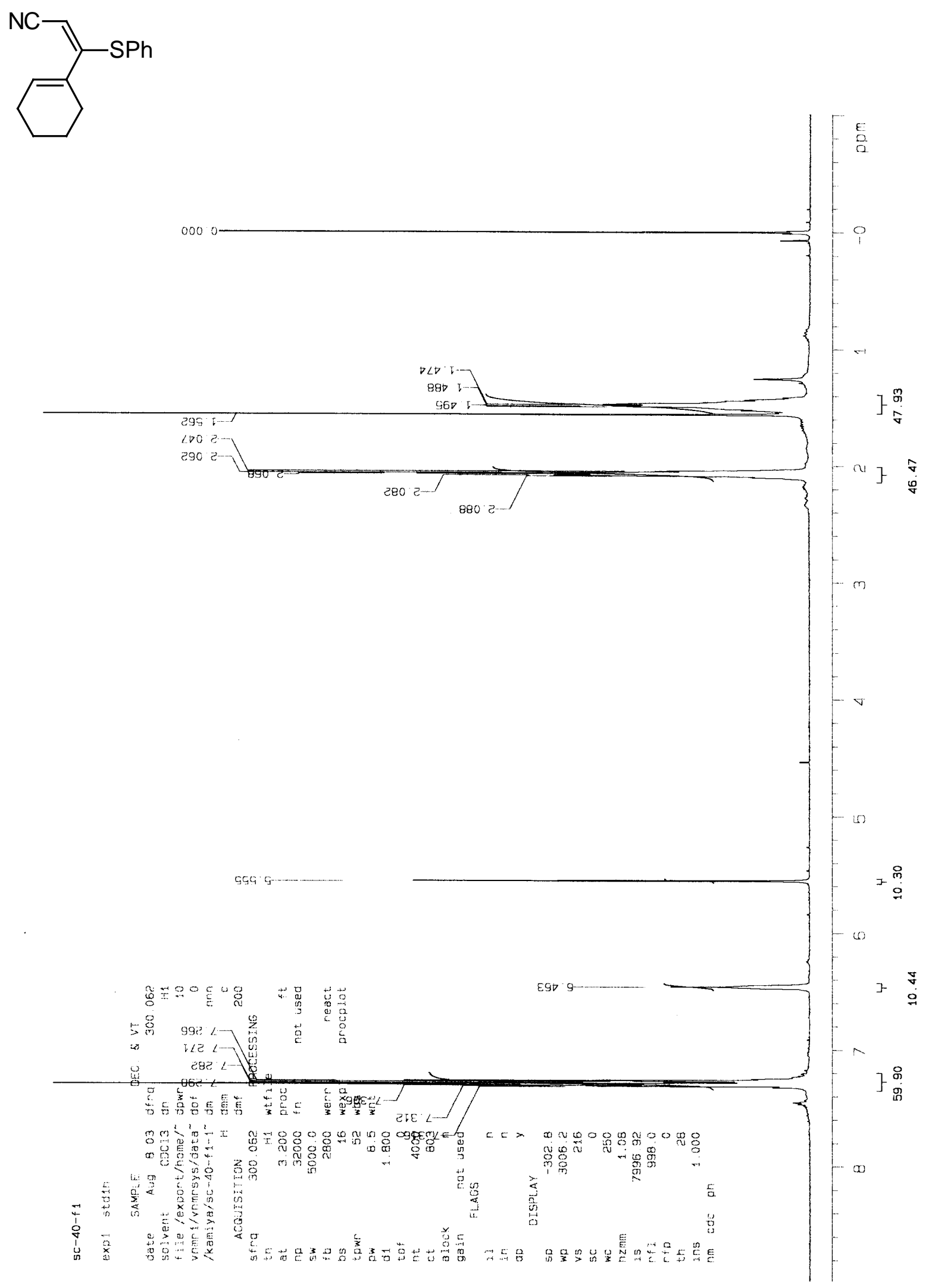



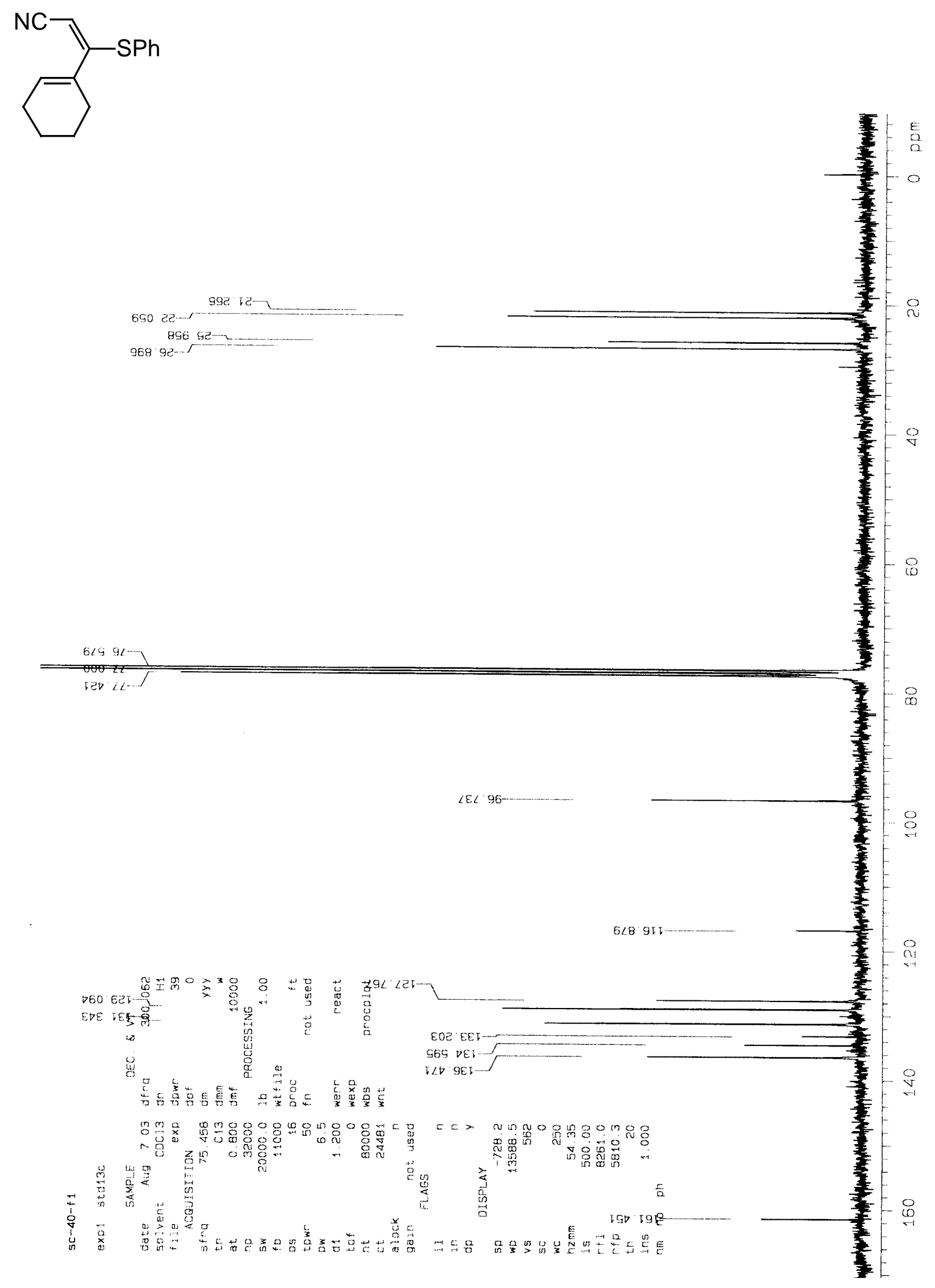
$\sum_{\mathrm{PPh}}^{\mathrm{CN}}$

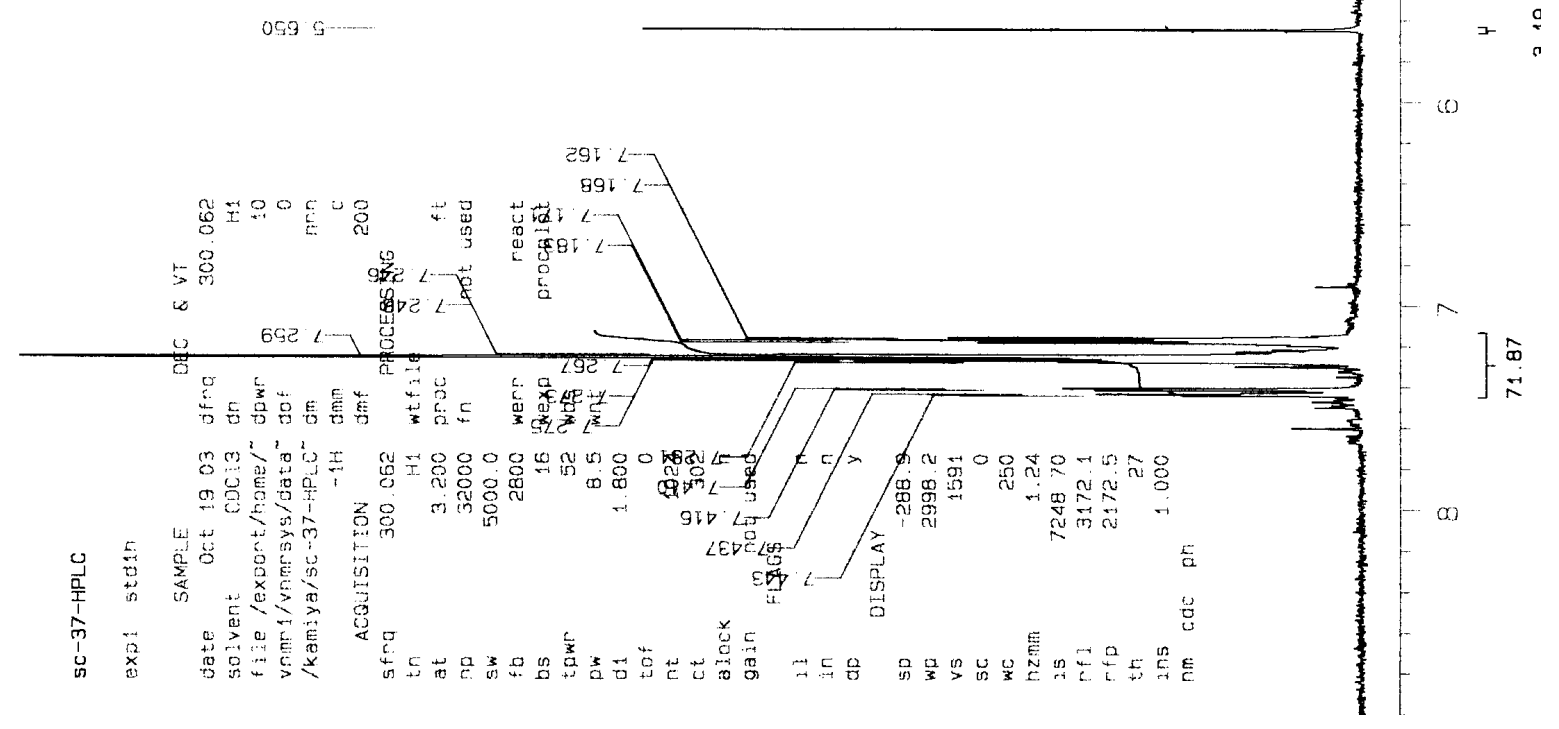



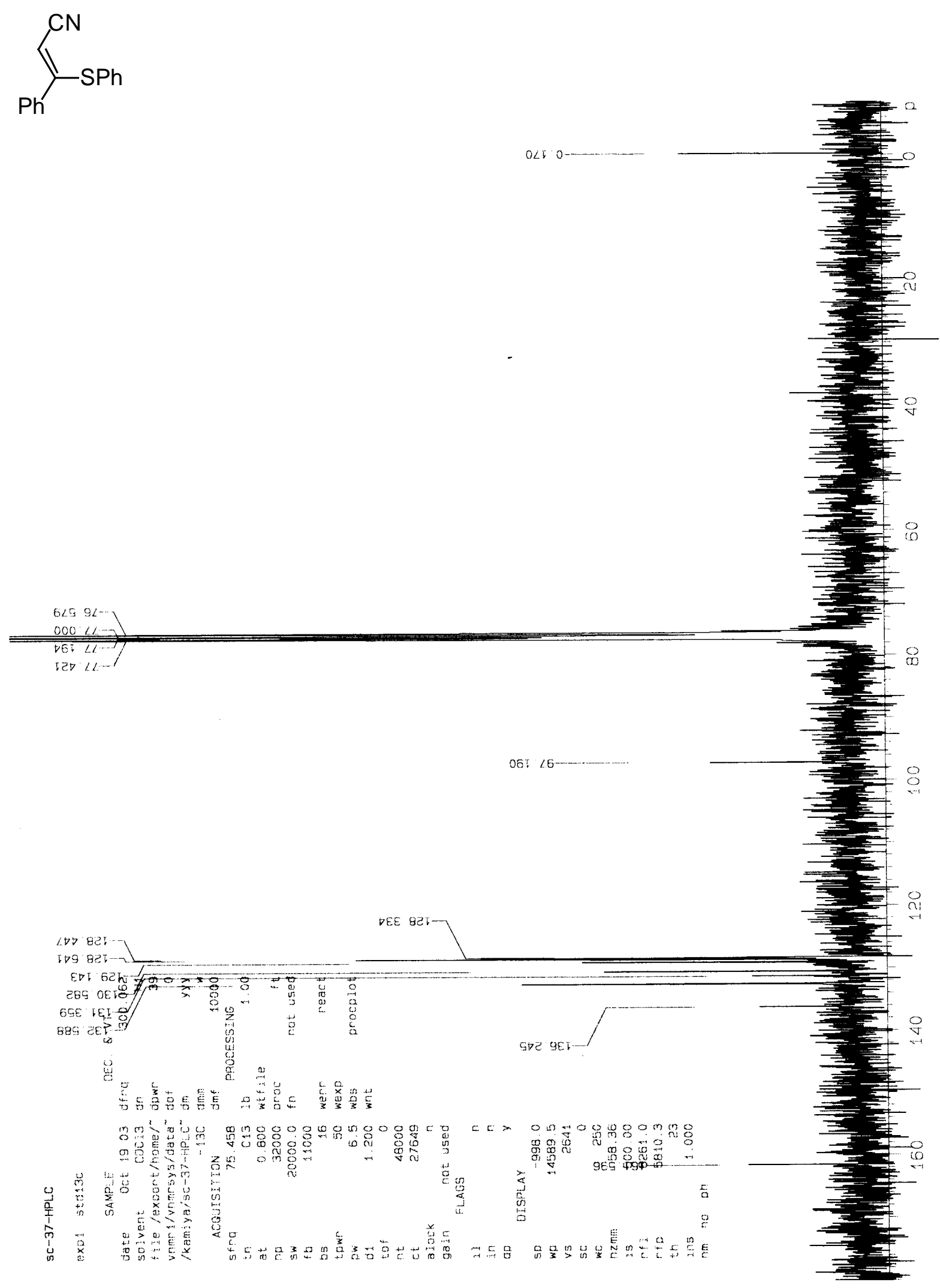


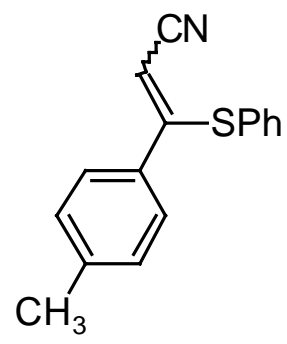

$0000 \cdots$

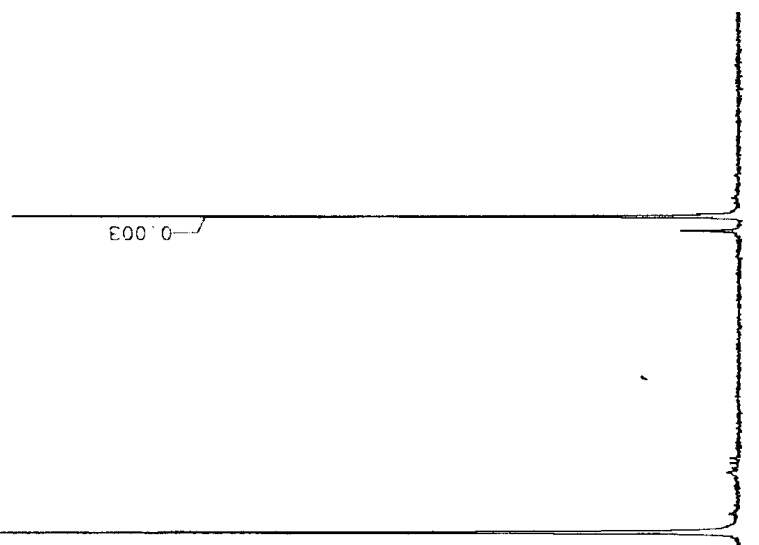

6DTt

982 ट

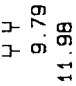

989

179.5

$090<$

$91021)$

टL $\angle$

$0851 \cdots$

$\angle 8 ;<1 / l$

LEI $\angle$

5t $2 \cdots$

2

$99 \mathrm{l}$

Ege L

$992<$

$08 \mathrm{C}:$

टЕE.

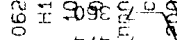

58

$\nabla \angle D \%$

w

I $60<1-1$

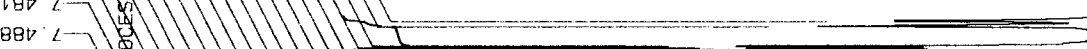

c.

5 L6t L

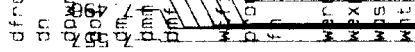

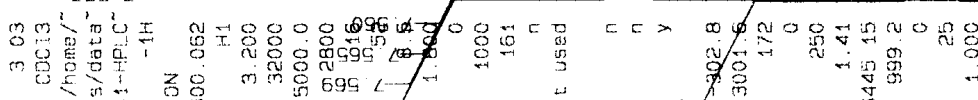

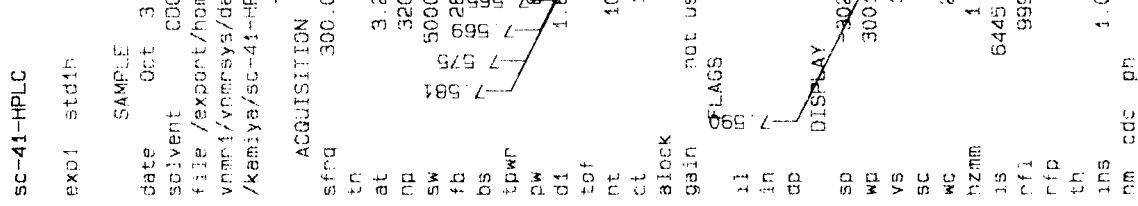

: $\Omega$

$=\begin{aligned} & \vec{\sigma} \\ & \text { पे }\end{aligned}$

$\omega$

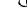

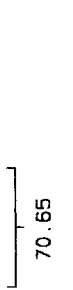

$\infty$ 
<smiles></smiles>

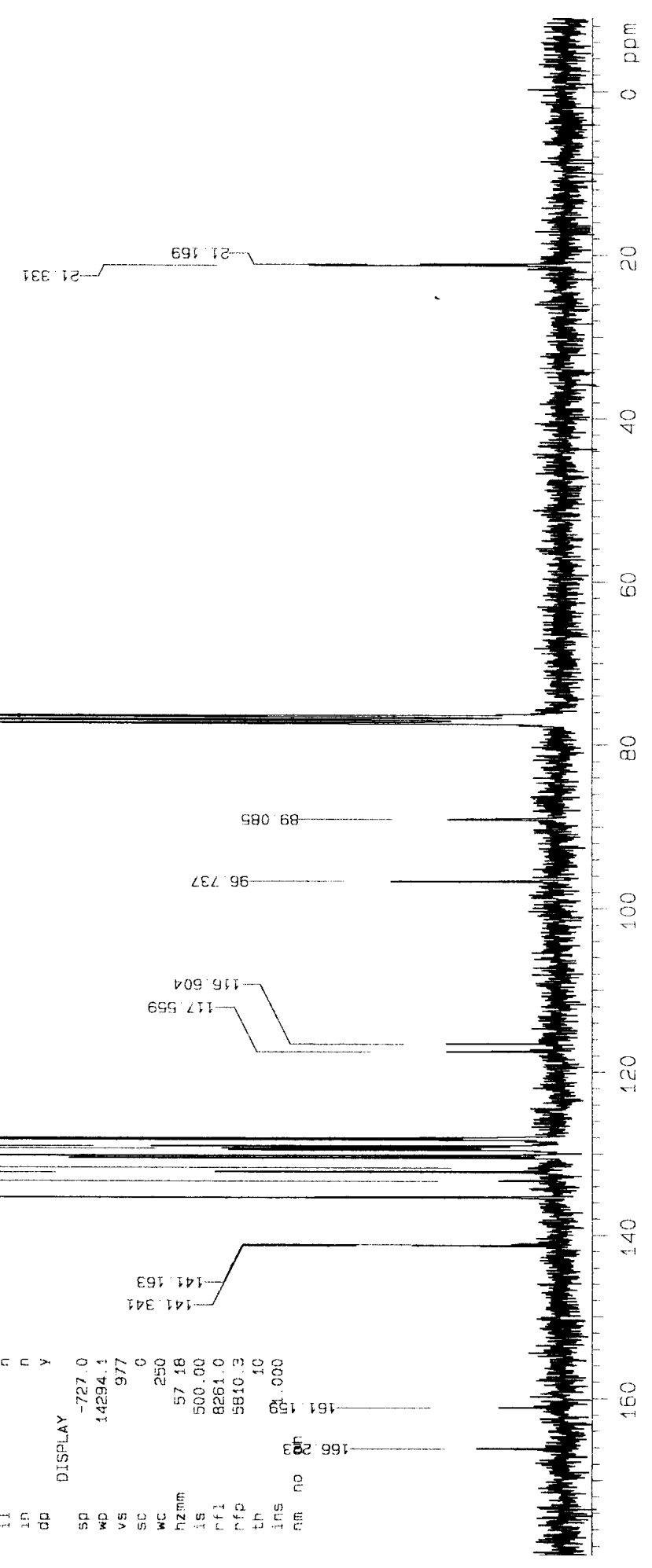

